T. H. KIM AND E. S. KIM

KODAI MATH. J.

18 (1995), 275-283

\title{
ITERATIVE FIXED POINTS OF NON-LIPSCHITZIAN SELF-MAPPINGS
}

\author{
TaE Hwa KIM* and EUn SuK Kim
}

\begin{abstract}
In this paper, we shall establish 1terative fixed points of non-Lipschitzian continuous self-mappings on Banach spaces with weak uniform normal structure.
\end{abstract}

\section{Introduction}

Let $C$ be a nonempty subset of a real Banach space $X$ and let $N$ be the set of natural numbers. A mapping $T: C \rightarrow C$ is said to be Lipschitzian if for each $n \in \boldsymbol{N}$, there exists a real number $k(n)$ such that

$$
\left\|T^{n} x-T^{n} y\right\| \leqq k(n)\|x-y\| \quad \text { for all } x, y \in C .
$$

In particular, $T$ is said to be asymptotically nonexpansive [7] if $\lim _{n \rightarrow \infty} k(n)=1$ and it is said to be nonexpansive if $k(n)=1$ for any $n \in \boldsymbol{N}$. We now consider a non-Lipschitzian self-mapping on $C$, that is to say, a mapping of weakly asymptotically nonexpansive type. We say that a mapping $T: C \rightarrow C$ is said to be weakly asymptotically nonexpansive type (simply, w.a.n.t.) on $C$ (see [10]) if, for each $x \in C$ and each bounded subset $D$ of $C$,

$$
\limsup _{n \rightarrow \infty}\left(\sup \left\{\left[\left\|T^{n} x-T^{n} y\right\|-\|x-y\|\right]: y \in D\right\}\right) \leqq 0 .
$$

Immediately, we can see that all mappings of w.a.n.t. include all mappings of asymptotically nonexpansive type (see [11]). In particular, if $T: C \rightarrow C$ is a Lipschitzian mapping with an additional condition, i.e., $\lim \sup _{n \rightarrow \infty} k(n) \leqq 1$ (see [12] and [14]), then it is obviously a continuous mapping of w.a.n.t. Further if $C$ is bounded, then any mapping of w.a.n.t. is asymptotically nonexpansive type.

1991 AMS Subject Classification. 47H09, 47H20.

Key words and phrases. Weakly asymptotically nonexpansive type, weak uniform normal structure and iterative fixed points.

* Supported by the Research Institute Attached to University Program, Korea Research Foundation, 1993.

Received February 18, 1994 ; revised July 21, 1994. 
In this paper, we first give new sharp expressions of the weak convergent sequence coefficient $W C S(X)$ of a non-Schur space $X$ according to G. L. Zhang [16]. Finally, we shall present an iterative fixed point of a non-Lipschitzian continuous self-mapping on a Banach space $X$ with weak uniform normal structure (see Theorem 3.3), which improves the result due to T.D. Benavides, G.L. Acedo and H.K. $\mathrm{Xu}$ [2].

\section{Expressions of the $W C S(X)$}

Let $X$ be a Banach space which is not Schur and let $\left\{x_{n}\right\}$ be a sequence of $X$. For $x \in X$, set $r\left(x,\left\{x_{n}\right\}\right)=\lim \sup _{n \rightarrow \infty}\left\|x_{n}-x\right\| . A\left(\left\{x_{n}\right\}\right)=\lim \sup _{n \rightarrow \infty}\left\{\| x_{i}-\right.$ $\left.x_{j} \|: i, j \geqq n\right\}$ and $r\left(\left\{x_{n}\right\}\right)=\inf \left\{r\left(x,\left\{x_{n}\right\}\right): x \in \overline{c o}\left(\left\{x_{n}\right\}\right)\right\}$ are called the asymptotic diameter of $\left\{x_{n}\right\}$ and the Chebyshev radius of $\left\{x_{n}\right\}$ relative to $\overline{c o}\left(\left\{x_{n}\right\}\right)$, respectively, where $\overline{c o}\left(\left\{x_{n}\right\}\right)$ denotes the closed convex hull of $\left\{x_{n}\right\}$. The weakly convergent sequence coefficient of $X$, denoted by $W C S(X)$, is the supremum of the set of all numbers $M$ with the property that for each weakly convergent sequence $\left\{x_{n}\right\}$ with asymptotic diameter $A\left(\left\{x_{n}\right\}\right)$, there is some $y \in \overline{c o}\left(\left\{x_{n}\right\}\right)$ such that $M \cdot \lim \sup _{n \rightarrow \infty}\left\|x_{n}-y\right\| \leqq A\left(\left\{x_{n}\right\}\right)$. Equivalently,

$$
W C S(X)=\inf \left\{A\left(\left\{x_{n}\right\}\right) / r\left(\left\{x_{n}\right\}\right)\right\},
$$

where the first infimum is taken over all sequences $\left\{x_{n}\right\}$ in $X$ which are weakly (not strongly) convergent (see [3] and [13]). It is well-known (see [3]) that if $W C S(X)>1$, then $X$ has weak normal structure. This means that any weakly compact convex subset $C$ of $X$ with $\operatorname{diam}(C)>0$ has a nondiametral point, i.e., an $x \in C$ such that

$$
\sup \{\|x-y\|: y \in C\}<\operatorname{diam}(C) .
$$

The coefficients $W C S(X)$ play important roles in fixed point theory (cf. [4], [9], [15]). A space $X$ such that $W C S(X)>1$ is said to have weak uniform normal structure.

Recently, G. L. Zhang [16] has proved the following improvement of expression of the $W C S(X)$ :

$$
W C S(X)=\sup \left\{M: x_{n} \rightarrow u \Rightarrow M \cdot \lim _{n} \sup _{n} x_{n}-u \| \leqq A\left(\left\{x_{n}\right\}\right)\right\},
$$

where " $\rightarrow$ " means the weak convergence.

For a sequence $\left\{x_{n}\right\}$ of a Banach space $X$, we set

$$
D\left(\left\{x_{n}\right\}\right):=\limsup _{m \rightarrow \infty}\left(\limsup _{n \rightarrow \infty}\left\|x_{n}-x_{m}\right\|\right) .
$$

We easily see that $D\left(\left\{x_{n}\right\}\right) \leqq A\left(\left\{x_{n}\right\}\right)$. However, $D\left(\left\{x_{n}\right\}\right) \neq A\left(\left\{x_{n}\right\}\right)$ in general. For example, consider the James' quasi-reflexive space $J$ consisting of all real sequences $x:=\left\{x_{n}\right\}=\sum_{n=1}^{\infty} x_{n} e_{n}$ for which $\lim _{n \rightarrow \infty} x_{n}=0$ and $\|x\|_{J}<\infty$, where 


$$
\|x\|_{J}=\sup \left\{\left[\left(x_{p_{1}}-x_{p_{2}}\right)^{2}+\cdots+\left(x_{p_{m-1}}-x_{p_{m}}\right)^{2}+\left(x_{p_{m}}-x_{p_{1}}\right)^{2}\right]^{1 / 2}\right\}
$$

and the supremum is taken over all choices of $m$ and $p_{1}<p_{2}<\cdots<p_{m}$. Then $J$ is a Banach space with the norm $\|\cdot\|_{J}$ and the sequence $\left\{e_{n}\right\}$ given by $e_{n}=$ $(0, \cdots, 0,1,0, \cdots)$ where the 1 is in the $n$th position, is a Schauder basis for $J$ (see [5]).

Take $x_{n}=e_{n}-e_{n+1}$ for each $n \in N$. Since $\left\|x_{n}\right\|_{J}=\sqrt{6}$ for each $n \in \boldsymbol{N}$, we have $x_{n} \in J$ and we now show that

$$
D\left(\left\{x_{n}\right\}\right)=2 \sqrt{3}<A\left(\left\{x_{n}\right\}\right)=2 \sqrt{5} .
$$

Indeed, for each fixed $n \in \boldsymbol{N}$, it is obvious that $\left\|x_{n}-x_{m}\right\|_{J}=2 \sqrt{3}$ for all $m \geqq n+3$ and so $D\left(\left\{x_{n}\right\}\right)=\lim \sup _{m \rightarrow \infty}\left(\lim \sup _{n \rightarrow \infty}\left\|x_{n}-x_{m}\right\|_{J}\right)=2 \sqrt{3}$. On the other hand, for each $k \in N$, if we take $n=k$ and $m=n+1 \geqq k$, then $\left\|x_{n}-x_{m}\right\|_{J}=2 \sqrt{5}$ and so

$$
\sup \left\{\left\|x_{n}-x_{m}\right\|_{J}: n, m \geqq k\right\}=2 \sqrt{5} \quad \text { for each } k \in N,
$$

which gives $A\left(\left\{x_{n}\right\}\right)=2 \sqrt{5}$.

We now give a sharp expression of $W C S(X)$ which improves the result due to G. H. Zhang [16; Theorem 1].

We begin with the following easy lemma.

LEMMA 2.1. If $z_{n}=y_{n} /\left\|y_{n}\right\|, \alpha:=\lim _{n \rightarrow \infty}\left\|y_{n}\right\| \neq 0$, then

$$
D\left(\left\{z_{n}\right\}\right)=\frac{1}{\alpha} D\left(\left\{y_{n}\right\}\right) .
$$

Proof. For each $n, m \in \boldsymbol{N}$, we easily get

$$
\begin{aligned}
\left\|z_{n}-z_{m}\right\| & =\left\|\frac{1}{\alpha}\left(y_{n}-y_{m}\right)+\left(\frac{1}{\left\|y_{n}\right\|}-\frac{1}{\alpha}\right) y_{n}-\left(\frac{1}{\left\|y_{m}\right\|}-\frac{1}{\alpha}\right) y_{m}\right\| \\
& \leqq \frac{1}{\alpha}\left\|y_{n}-y_{m}\right\|+\left|\frac{1}{\left\|y_{n}\right\|}-\frac{1}{\alpha}\right| \cdot\left\|y_{n}\right\|+\left|\frac{1}{\left\|y_{m}\right\|}-\frac{1}{\alpha}\right| \cdot\left\|y_{m}\right\| .
\end{aligned}
$$

Taking at first lim sup $\operatorname{sum}_{m \rightarrow \infty}$ and next $\lim \sup _{n \rightarrow \infty}$ in both sides, we obtain

$$
D\left(\left\{z_{n}\right\}\right) \leqq \frac{1}{\alpha} D\left(\left\{y_{n}\right\}\right) .
$$

The converse inequality is similarly obtained.

LEMMA 2.2. Let $M>0$. Then the following statements are equivalent:

(a) $M \cdot \limsup _{n \rightarrow \infty}\left\|x_{n}-x\right\| \leqq A\left(\left\{x_{n}\right\}\right)$ for any $x_{n} \rightarrow x$ (not strongly convergent).

(b) $M \cdot \limsup _{n \rightarrow \infty}\left\|x_{n}^{\prime}-x^{\prime}\right\| \leqq D\left(\left\{x_{n}^{\prime}\right\}\right)$ for any $x_{n}^{\prime} \rightarrow x^{\prime}$ (not strongly convergent).

Proof. Since $D\left(\left\{x_{n}\right\}\right) \leqq A\left(\left\{x_{n}\right\}\right)$, it is obvious that (b) $\Rightarrow(\mathrm{a})$. 
To show $(\mathrm{a}) \Rightarrow(\mathrm{b})$, let $x_{n}^{\prime} \rightarrow x^{\prime}$ (not strongly convergent) and $\alpha:=\lim \sup _{n \rightarrow \infty}$ $\left\|x_{n}^{\prime}-x^{\prime}\right\| \neq 0$. Then we can choose a subsequence $\left\{x_{m}^{\prime}\right\}$ of $\left\{x_{n}^{\prime}\right\}$ such that $\alpha=$ $\lim _{m \rightarrow \infty}\left\|x_{m}^{\prime}-x^{\prime}\right\|$. Setting $z_{m}:=\left(x_{m}^{\prime}-x^{\prime}\right) / \alpha$, we have $z_{m} \rightarrow 0$ and $\left\|z_{m}\right\| \rightarrow 1$, by using a diagonal method as in [1], we can choose a subsequence $\left\{z_{m_{k}}\right\}$ of $\left\{z_{m}\right\}$ such that $\lim _{k, l \rightarrow \infty k \neq l}\left\|z_{m_{k}}-z_{m_{l}}\right\|$ exists. Then (a) and Lemma 2.1 yield that

$$
\begin{aligned}
M & =M \cdot \lim _{k \rightarrow \infty}\left\|z_{m_{k}}\right\| \leqq A\left(\left\{z_{m_{k}}\right\}\right)=D\left(\left\{z_{m_{k}}\right\}\right) \\
& \leqq D\left(\left\{z_{m}\right\}\right)=\frac{1}{\alpha} D\left(\left\{x_{m}^{\prime}\right\}\right) \leqq \frac{1}{\alpha} D\left(\left\{x_{n}^{\prime}\right\}\right)
\end{aligned}
$$

and hence $M \cdot \lim \sup _{m \rightarrow \infty}\left\|x_{m}^{\prime}-x^{\prime}\right\|=M \alpha \leqq D\left(\left\{x_{n}^{\prime}\right\}\right)$, which completes the proof.

As a direct consequence of Lemma 2.2 and (2.1), we can obtain the following

THEOREM 2.3.

$$
W C S(X)=\sup \left\{M: x_{n} \rightarrow u \Rightarrow M \cdot \lim \sup _{n \rightarrow \infty}\left\|x_{n}-u\right\| \leqq D\left(\left\{x_{n}\right\}\right)\right\} .
$$

As a direct consequence of Theorem 2.3, by using a similar method as in G. H. Zhang [16], we can easily obtain the following expressions of $W C S(X)$.

$$
\begin{aligned}
W C S(X) & =\inf \left\{\frac{D\left(\left\{x_{n}\right\}\right)}{r\left(u,\left\{x_{n}\right\}\right)}:\left\{x_{n}\right\} \text { weakly (not strongly) converges to } u\right\} \\
& =\inf \left\{D\left(\left\{x_{n}\right\}\right):\left\{x_{n}\right\} \subset S(X) \text { and } x_{n} \rightarrow 0\right\} \\
& =\inf \left\{D\left(\left\{x_{n}\right\}: x_{n} \rightarrow 0 \text { and } \lim _{n \rightarrow \infty}\left\|x_{n}\right\|=1\right\}\right.
\end{aligned}
$$

where $S(X)$ denotes the unit sphere of $X$, i.e., $S(X)=\{x \in X:\|x\|=1\}$.

\section{Iterative fixed points of non-Lipschitzian mappings}

Let $C$ be a nonempty subset of a Banach space $X$. In this section, we recall that a mapping $T: C \rightarrow C$ is said to be weakly asymptotically nonexpansive type (simply, w.a.n.t.) on $C$ (see [10]) if, for each $x \in C$ and each bounded subset $D$ of $C$,

$$
\limsup _{n \rightarrow \infty}\left(\sup \left\{\left[\left\|T^{n} x-T^{n} y\right\|-\|x-y\|\right]: y \in D\right\}\right) \leqq 0 .
$$

Here we give an example of a continuous mapping which is of w.a.n.t. and not Lipschitz.

Example. Let $X=\boldsymbol{R}, C=[0, \infty)$ and let $|k|<1$. For each $n \in \boldsymbol{N}_{0}=\boldsymbol{N} \cup\{0\}$, we define

$$
T^{n+1} x= \begin{cases}k T^{n} x\left|\sin \frac{1}{T^{n} x}\right|, & \text { if } \quad x \in C-Z\left(T^{n}\right) ; \\ 0, & \text { if } \quad x \in Z\left(T^{n}\right) .\end{cases}
$$


We denote by $Z\left(T^{n}\right)$ the set of all zeros of $T^{n}$ and by $T^{0}$ the identy mapping $I$ on $C$. We see that $Z(T)=\{1 / m \pi: m \in N\} \cup\{0\}, Z\left(T^{n}\right)=Z\left(T^{n-1}\right) \cup\{z \in C$ : $T^{n-1} z=(1 / m \pi)$ for some $\left.m \in N\right\}$ for $n \in N$, and $\left\{Z\left(T^{n}\right): n \in N\right\}$ is nondecreasing. Since $\left\{T^{n} x\right\}$ converges uniformly to zero on any closed bounded interval of $C$, $T \equiv T^{1}: C \rightarrow C$ is really a continuous mapping of w.a.n.t. Indeed, for each $x \in C$ and each bounded subset $D$ of $C$, there is a closed bounded interval $I_{D}$ containing $D$. For each $n \in N$, the map $h_{n}(y)=\left|T^{n} x-T^{n} y\right|-|x-y|$ achieves its maximum on $I_{D}$, say $y_{n} \in I_{D}$. Then,

$$
\begin{aligned}
& \limsup _{n \rightarrow \infty}\left\{\left|T^{n} x-T^{n} y\right|-|x-y|: y \in I_{D}\right\} \\
= & \limsup _{n \rightarrow \infty}\left(\left|T^{n} x-T^{n} y_{n}\right|-\left|x-y_{n}\right|\right) \\
\leqq & \lim _{n \rightarrow \infty}\left|T^{n} x-T^{n} y_{n}\right|-\liminf _{n \rightarrow \infty}\left|x-y_{n}\right| \\
= & -\liminf _{n \rightarrow \infty}\left|x-y_{n}\right| \leqq 0 .
\end{aligned}
$$

Therefore $T: C \rightarrow C$ is of w.a.n.t. However, it is obviously not Lipschitz.

Recall that $T: C \rightarrow C$ is said to be asymptotically regular on $C$ if

$$
\lim _{n \rightarrow \infty}\left\|T^{n} x-T^{n+1} x\right\|=0 \quad \forall x \in C .
$$

Definition 3.1. A mapping $T: C \rightarrow X$ is said to be weakly demicompact if whenever $\left\{x_{n}\right\} \subset C$ is a bounded sequence and $\left\{x_{n}-T x_{n}\right\}$ is a convergent sequence, then there exists a subseqence $\left\{x_{n_{k}}\right\}$ of $\left\{x_{n}\right\}$ which is weakly convergent.

It is clear that every demicompact mappings are weakly demicompact, and also that if $C$ is weakly compact, then $T: C \rightarrow X$ is weakly demicompact.

LEMMA 3.2. Let $C$ be a nonempty closed convex separable subset of a Banach space $X$ and let $T: C \rightarrow C$ be a mapping of w.a.n.t. Let $x_{0} \in C$ such that $\left\{T^{n} x_{0}\right\}$ is bounded. If $T$ is weakly demicompact and asymptotically regular on $C$, then there exists a subsequence $\left\{n_{j}\right\}$ of positive integers such that

$$
\left\{T^{n}, x\right\} \text { converges weakly for every } x \in C \text {. }
$$

Proof. Since $T$ is a mapping of w.a.n.t., it is obvious that for each $x \in C$, $\left\{T^{n} x\right\}$ is bounded. For each $x \in C$, we set $x_{n}=T^{n} x$ for $n \in \boldsymbol{N}$. Since $T$ is asymptotically regular on $C$, by the weak demicompactness of $T$, there exists a subsequence $\left\{x_{n_{k}}\right\}$ of $\left\{x_{n}\right\}$ which converges weakly.

Let $E$ be a countable dense subset of $C$. By using a usual diagonal method, we can choose a subsequence $\left\{n_{j}\right\}$ of $N$ such that $\left\{T^{n} z\right\}$ converges weakly to $u_{z} \in C$ for every $z \in E$. Given $\varepsilon>0, x \in C$ and $x *(\neq 0) \in X^{*}$, where $X^{*}$ is a dual space of $X$, there is a $z \in E$ such that $\|x-z\|<\varepsilon / 3\|x *\|$. Since $T$ is a mapping of w.a.n.t., it follows that, for each $x, z \in C$ and for any subsequence $\left\{n_{i}\right\}$ of $\boldsymbol{N}$, 


$$
\limsup _{\imath \rightarrow \infty}\left\|T^{n_{2}} x-T^{n_{2}} z\right\| \leqq\|x-z\|,
$$

in particular, there exists $j_{0}^{\prime} \in \boldsymbol{N}$ such that

$$
\sup _{\jmath \geq j_{0}^{\prime}}\left\|T^{n} \jmath x-T^{n} z\right\| \leqq\|x-z\|+\frac{\varepsilon}{3\|x *\|}<\frac{2 \varepsilon}{3\|x *\|} .
$$

On the other hand, since $\left\{T^{n_{j}} z\right\}$ converges weakly to $u_{z} \in C$, there also exists $j_{0}^{\prime \prime} \in \boldsymbol{N}$ such that

$$
\left\|x *\left(T^{n} z-u_{z}\right)\right\|<\frac{\varepsilon}{3} \quad \text { for } \quad j \geqq j_{0}^{\prime \prime},
$$

By taking $\jmath_{0}=\max \left\{j_{0}^{\prime}, j_{0}^{\prime \prime}\right\}$, we obtain that, for $j \geqq \jmath_{0}$,

$$
\left\|x *\left(T^{n}, x-u_{z}\right)\right\| \leqq\|x *\|\left\|T^{n}, x-T^{n}, z\right\|+\left\|x *\left(T^{n}, z-u_{z}\right)\right\|<\varepsilon .
$$

which completes the proof.

If $C$ is a closed convex subset of a Banach space $X$ and if $T: C \rightarrow C$ is a mapping, we can easily construct a separable subspace $X_{\infty}$ of $X$ and a closed convex subset $C_{\infty}$ of $X_{\infty}$ which is $T$-invariant. This shows that in many cases, i.e., when the other assumptions on $C$ are inherited by $C_{\infty}$, it suffices to formulate fixed point problems in a separable setting (see [7; pp. 35-36]).

By using an iterative method, we show a fixed point theorem of a continuous self-mapping of w.a.n.t. on a Banach space $X$ with weak uniform normal structure, i.e., $W C S(X)>1$. We employ the method of the proof of [2].

TheOREM 3.3. Suppose $X$ is a Banach space such that $W C S(X)>1, C$ is a nonempty closed convex subset of $X$, and a continuous mapping $T: C \rightarrow C$ of w.a.n.t. is weakly demicompact. Suppose in addition that $T$ is asymptotically regular on $C$ and $\left\{T^{n} x_{0}\right\}$ is bounded for some $x_{0} \in C$. Then $T$ has an iterative fixed point in $C$.

Proof. By above argument, we may assume that $C$ is separable. By Lemma 3.2, we can choose a subsequence $\left\{n_{j}\right\}$ of positive integers such that

$$
\left\{T^{n}, x\right\} \quad \text { converges weakly for every } x \in C \text {. }
$$

Now we can construct a sequence $\left\{x_{n}\right\}$ in $C$ in the following way:

$$
\begin{aligned}
& x_{0} \in C \quad \text { arbitrary } \\
& x_{m}=w-\lim _{\jmath \rightarrow \infty} T^{n} x_{m-1}, \quad \forall m \geqq 1 .
\end{aligned}
$$

Note that the asymptotic regularity of $T$ on $C$ ensures that

$$
x_{m}=w-\lim _{j \rightarrow \infty} T^{n_{j}+p} x_{m-1}, \quad \forall p \geqq 0 .
$$

We now show that $\left\{x_{m}\right\}$ converges strongly to a fixed point of $T$. To this 
end, for each integer $m \geqq 0$,

$$
B_{m}:=\limsup _{\jmath \rightarrow \infty}\left\|T^{n} x_{m}-x_{m+1}\right\| .
$$

Then, by Theorem 2.3, we have

$$
B_{m} \leqq \frac{1}{W C S(X)} D\left(\left\{T^{n}, x_{m}\right\}\right) .
$$

Since $T$ is a mapping of w.a.n.t., for each fixed $m \in N$ and bounded subset $D_{m}=\left\{T^{n} x_{m}: n \in \boldsymbol{N}\right\}$ of $C$

$$
\limsup _{j \rightarrow \infty}\left\{\sup \left[\left\|T^{n}, x_{m}-T^{n} \boldsymbol{z}\right\|-\left\|x_{m}-z\right\|\right]: z \in D_{m}\right\} \leqq 0,
$$

and by asymptotic regularity of $T$ on $C$, it follows that, for each $j \in \boldsymbol{N}$ and $p \geqq 0$,

$$
\limsup _{\imath \rightarrow \infty}\left\|T^{n_{i}+p} u-T^{n} v v\right\|=\limsup _{\imath \rightarrow \infty}\left\|T^{n_{\imath}} u-T^{n} v\right\| \quad \forall u, v \in C .
$$

Replacing $p$ and $u, v$ in (3.3) by $n$, and $x_{m}$ respectively, it follows that

$$
\begin{aligned}
& D\left(\left\{T^{n}, x_{m}\right\}\right)=\limsup _{\jmath \rightarrow \infty}\left(\limsup _{\imath \rightarrow \infty}\left\|T^{n_{\imath}} x_{m}-T^{n}, x_{m}\right\|\right) \\
& =\limsup _{\jmath \rightarrow \infty}\left(\limsup _{\imath \rightarrow \infty}\left\|T^{n_{i}+n_{\jmath}} x_{m}-T^{n_{\imath}} x_{m}\right\|\right) .
\end{aligned}
$$

Noting also that, for each $j \in \boldsymbol{N}$,

$$
\begin{aligned}
& \limsup _{\imath \rightarrow \infty}\left\|T^{n_{i}+n_{J}} x_{m}-T^{n_{J}} x_{m}\right\| \\
& \leqq \sup _{\imath \in N}\left[\left\|T^{n_{\jmath}} x_{m}-T^{n_{j}}\left(T^{n_{\imath}} x_{m}\right)\right\|-\left\|x_{m}-T^{n_{\imath}} x_{m}\right\|\right] \\
& \quad+\limsup _{\imath \rightarrow \infty}\left\|x_{m}-T^{n_{\imath}} x_{m}\right\| \\
& \leqq \\
& \quad \sup _{z \in D}\left[\left\|T^{n_{\jmath}} x_{m}-T^{n_{\jmath}} z\right\|-\left\|x_{m}-z\right\|\right] \\
& \quad+\limsup _{\imath \rightarrow \infty}\left\|x_{m}-T^{n_{\imath}} x_{m}\right\|,
\end{aligned}
$$

it follows from (3.2) that

$$
D\left(\left\{T^{n}, x_{m}\right\}\right) \leqq \limsup _{i \rightarrow \infty}\left\|x_{m}-T^{n_{2}} x_{m}\right\| .
$$

On the other hand, by the $w-$ l.s.c. of the norm of $X$ and with the same method as before, we easily obtain

$$
\begin{aligned}
& \limsup _{\imath \rightarrow \infty}\left\|x_{m}-T^{n_{\imath}} x_{m}\right\| \leqq \limsup _{\imath \rightarrow \infty}\left(\liminf _{\jmath \rightarrow \infty}\left\|T^{n_{\imath}} x_{m}-T^{n} \jmath x_{m-1}\right\|\right) \\
& \leqq \limsup _{\imath \rightarrow \infty}\left(\limsup _{j \rightarrow \infty}\left\|T^{n_{\imath}} x_{m}-T^{n_{j}+n_{\imath}} x_{m-1}\right\|\right) \\
& \leqq \limsup _{j \rightarrow \infty}\left\|x_{m}-T^{n_{\jmath}} x_{m-1}\right\|=B_{m-1},
\end{aligned}
$$


which immediately gives that $D\left(\left\{T^{n}, x_{m}\right\}\right) \leqq B_{m-1}$. Hence, by (3.1),

$$
B_{m} \leqq \frac{1}{W C S(X)} B_{m-1}
$$

and since $W C S(X)>1$, we have $\lim _{m \rightarrow \infty} B_{m}=0$. Now using the $w-$ l.s.c. of the norm of $X$ and (3.4) again, we deduce that

$$
\begin{aligned}
\left\|x_{m}-x_{m+1}\right\| & \leqq \limsup _{\imath \rightarrow \infty}\left\|x_{m}-T^{n_{\imath}} x_{m}\right\|+\limsup _{\imath \rightarrow \infty}\left\|T^{n_{\imath}} x_{m}-x_{m+1}\right\| \\
& \leqq B_{m-1}+B_{m},
\end{aligned}
$$

which implies that $\left\{x_{m}\right\}$ is Cauchy. Let $v:=\lim _{m \rightarrow \infty} x_{m}$. Then, for each $\jmath \in \boldsymbol{N}$,

$$
\left\|v-T^{n}, v\right\| \leqq\left\|v-x_{m+1}\right\|+\left\|x_{m+1}-T^{n}, x_{m}\right\|+\left\|T^{n}, x_{m}-T^{n}, v\right\| .
$$

Since $T$ is of w.a.n.t., by taking the $\lim \sup _{j \rightarrow \infty}$ in both sides we obtain that

$$
\limsup _{j \rightarrow \infty}\left\|v-T^{n} \jmath\right\| \leqq\left\|v-x_{m+1}\right\|+B_{m}+\left\|x_{m}-v\right\| \rightarrow 0
$$

as $m \rightarrow \infty$. Hence $T^{n}, v \rightarrow v$ and $T v=v$ by the continuity and asymptotic regularity of $T$ at $v$. This completes the proof.

COROLlary 3.4. Suppose $X$ is a Banach space such that $W C S(X)>1, C$ is a nonempty weakly compact convex subset of $X$, and $T: C \rightarrow C$ is a continuous mapping of asymptotically nonexpansive type. Suppose in addition that $T$ is asymptotically regular on $C$. Then $T$ has an iterative fixed point in $C$.

Finally, as a direct consequence of Corollary 3.4, we give an iterative fixed point for a nonexpansive mappng on a Banach space. Let $T: C \rightarrow C$ be a nonexpansive mapping. For a fixed $\lambda \in(0,1)$, we set

$$
S_{\lambda}:=\lambda I+(1-\lambda) T,
$$

where $I$ is the identity operator of $X$. Then it is obvious that $S_{\lambda}: C \rightarrow C$ is also nonexpansive with the same fixed point set of $T$. Moreover, it is wellknown (see [6]) that $S_{\lambda}$ is asymptotically regular on $C$. Therefore, we obtain the following result due to T.D. Benavides, G. L. Acedo and H. K. Xu [2].

Corollary 3.5. Suppose $X$ is a Banach space such that $W C S(X)>1, C$ is a nonempty weakly compact convex subset of $X$, and $T: C \rightarrow C$ is a nonexpansive mapping. Then $T$ has an iterative fixed point in $C$.

\section{REFERENCES}

[1] T.D. Benavides, Some properties of the set and ball measures of noncom. pactness and applications, J. London Math. Soc., 34 (1986), 120-128. 
[2] T.D. Benavides, G.L. Acedo And H.K. XU, Weak uniform normal structure and iterative fixed points of nonexpansive mappings, Colloq. Math. (to appear).

[3] W.L. Bynum, Normal structure coefficient for Banach spaces, Pacific J. Math., 86 (1980), 427-436.

[4] E. CAsini and E. Maluta, Fixed points of uniformly Lipschitzian mappings in spaces with uniformly normal structure, Nonlinear Anal., 9 (1985), 103-108.

[5] J. Diestel, Sequences and Series in Banach Spaces, Graduate Texts in Math., 92, Springer-Verlag, New York, 1984.

[6] M. EdELSTEIN AND R.C. OB́RIEN, Nonexpansive mappings, asymptotic regularity, and successive approximations, J. London Math. Soc., 17 (1978), 547-554.

[7] K. Goebel AND W.A. KiRK, A fixed point theorem for asymptotically nonexpansive mappings, Proc. Amer. Math. Soc., 35 (1972), 171-174.

[8] K. Goebel AND W.A. KIRK, Topics in metric fixed point theory, Cambridge Univ. Press., 1990, pp. 34-35, 73-74.

[9] A. Jiménez-Melado, Stability of weak normal structure in James quasi reflexive space, Bull. Austral. Math. Soc., 46 (1992), 367-372.

[10] T.H. KIM, Nonlinear ergodic theorems of almost-orbits of non-Lipschitzian semigroups, Kodai Math. J., 15 (1992), 296-309.

[11] W.A. KIRK, Fixed point theorems for non-Lipschitzian mappings of asymptotically nonexpansive type, Israel J. Math., 17 (1976), 339-346.

[12] W. A. KIRK AND R. TORREJON, Asymptotically nonexpansive semigroups in Banach spaces, Nonlinear Anal., 3 (1979), 111-121.

[13] T.C. Lim, On the normal structure coefficient and the bounded sequence coefficient, Proc. Amer. Math. Soc., 88 (1983), 262-264.

[14] W. TAKahashi AND P.J. Zhang, Asymptotic behavior of almost-orbits of semigroups of Lipschitzian mappings in Banach spaces, Kodai Math. J., 11 (1988), $129-140$.

[15] D. Tingley, The normal structure of James quasi reflexive space, Bull. Austral. Math. Soc., 42 (1990), 95-100.

[16] G.L. ZHANG, Weakly convergent sequence coefficient of product space, Proc. Amer. Math. Soc., 117 (1993), 637-643.

Department of Applied Mathematics

National Fisheries University of Pusan

PUSAN 608-737

KOREA 